\title{
SOBRECARGA, APOYO SOCIAL Y AUTOCUIDADO EN CUIDADORES INFORMALES
}

\author{
BURDEN, SOCIAL SUPPORT AND SELF-CARE IN \\ INFORMAL CAREGIVERS
}

\author{
Karina Espinoza Miranda* \\ Viviane Jofre AravenA ${ }^{* *}$
}

\begin{abstract}
RESUMEN
Objetivo: Conocer el nivel de sobrecarga, el apoyo social percibido, la capacidad de agencia de autocuidado y otros factores relacionados, en cuidadores/as informales de pacientes dependientes de la comuna de Tomé, Chile. Material y método: estudio descriptivo. Muestra probabilística, sistemática y estratificada estuvo compuesta por 30 cuidadores/as informales, a quienes se les aplicaron cuatro instrumentos por medio de visitas domiciliarias: cuestionario de evaluación biosociodemográfica del cuidador informal principal, escala de sobrecarga de Zarit, Escala Multidimensional de Apoyo Social Percibido de Zimet y Escala de Apreciación de Agencia de Autocuidado (ASA). Resultados: Los cuidadores/as informales son: mujeres, adultas, con pareja, con educación básica incompleta, sin trabajo ni participación social y con percepción económica insuficiente, dedicando gran parte del día a cuidar, sin ayuda de terceros, con niveles de sobrecarga leve, escasa percepción de apoyo social y desarrollo de moderada capacidad de agencia de autocuidado. Conclusión: Los resultados emanados de esta investigación permiten colaborar en la generación de políticas públicas que apoyen a este grupo de interés vulnerable desde el punto de vista social y sanitario.
\end{abstract}

Palabras clave: Sobrecarga, apoyo social, autocuidado, cuidadores.

\begin{abstract}
Objective: Knowing the level of burden, perceived social support, the ability of self-care agency and other factors related, in informal caregiver of dependant patients in the city of Tomé, Chile. Methods: Descriptive study. The systematic stratified probabilistic sample consisted of 30 informal caregivers to whom four instruments were applied through home visits: bio sociodemographic questionnaire for the principal informal caregiver, Zarit Burden Scale, Multidimensional Scale of Perceived Social Support by Zimet and Appraisal of Self-care Agency Scale (ASA). Results: the informal caregivers are adult women, with partners, with incomplete primary education, no work or social participation and insufficient economic perception, devoting much of their day to care, without assistance from others, with levels of mild overload, poor perception of social support and moderate capacity of self-care. Conclusions: Results emerging from this research can cooperate in the generation of public policies to support this vulnerable group of interest from a social and health standpoint.
\end{abstract}

Key words: Burden, social support, self-care, caregivers.

Fecha recepción: 26/12/11 Fecha aceptación: 04/09/12

\footnotetext{
* Enfermera. Profesora Departamento de Enfermería, Facultad de Medicina, Universidad de Concepción, Chile. Email: kespinozam@udec.cl.

${ }^{* *}$ Enfermera. Profesora Departamento de Enfermería, Universidad de Concepción, Chile. Email: vjofre@udec.cl
} 


\section{INTRODUCCIÓN}

La evolución de la salud de la población chilena ha pasado de elevados niveles de morbimortalidad asociada a enfermedades infectocontagiosas durante los años 60, a un predominio de enfermedades cardiovasculares en la década de los 90. Hoy la mortalidad general en Chile está determinada fundamentalmente por enfermedades del sistema circulatorio, con un 27,1\% al año 2009, seguida por los tumores malignos en un 24,6\% (1). La esperanza de vida ha alcanzado los 78,3 años como promedio (2), el cual se estima aumente a 79,1 años en el periodo 20102025 (3). Sin embargo, este aumento de la esperanza de vida se encuentra acompañado por la presencia de patologías crónicas cardiovasculares, lo que ha generado un envejecimiento de la población, asociado a discapacidad y deterioro en la calidad de vida.

Según el Primer Estudio de Discapacidad en Chile, el $12,5 \%$ de la población total chilena vive con algún grado de discapacidad. El 62,9\% de éstos señala que su discapacidad la generó una enfermedad crónica y el 34,6\% de los hogares del país poseen un integrante con discapacidad, concentrándose fundamentalmente en los adultos mayores (4).

Este cambio demográfico, en forma conjunta con la alta demanda asistencial de los sistemas hospitalarios chilenos, ha llevado a altas precoces, delegando la responsabilidad de los cuidados a la familia, así surge el "cuidador informal", aquel que atiende en su hogar, y sin recibir remuneración, a familiares que tienen un daño crónico o discapacitante que les impide desenvolverse diariamente con independencia (5).

Se entiende como "cuidado informal" a "un sistema informal de prestación de cuidados no remunerado a personas dependientes, por parte de familiares, amigos $\mathrm{u}$ otras personas, conformando un verdadero sistema oculto de cuidados" (5), caracterizado por la ausencia de organización explícita (6). Hay ciertos rasgos que caracterizan el cuidado informal y que afectan directamente su visibilidad y reconocimiento social: se trata de un trabajo no remunerado, sin precio en el mercado, y esto se confunde con una carencia de valor. El cuidado se basa en relaciones afectivas y de parentesco, perteneciente al terreno de lo privado; se trata de "asuntos de familia", en los que el resto de la sociedad no se implica. Se desarrolla en el ámbito doméstico y, como tal, queda oculto a la arena pública. Finalmente, es una función adscrita a las mujeres como parte del rol de género: es "cosa de mujeres" (7).

Sin duda el cuidar también implica dar apoyo emocional y social (7), entendido éste como la provisión instrumental y/o expresiva, real y percibida, aportada por la comunidad, redes sociales y amigos íntimos (8).

Se estima que hasta en un $88 \%$ del total de cuidados prestados corresponde a cuidados de tipo informal (9). El 68\% de las personas con discapacidad recibe apoyo por parte de su familia (10).

Las condiciones en que se desarrollan las actividades diarias del cuidador/a informal, lo hace un ser vulnerable desde el punto de vista sanitario. La sobrecarga del cuidador comprende un conjunto de problemas físicos, mentales y socioeconómicos que sufren los cuidadores de personas enfermas, afectando sus actividades de ocio, relaciones sociales, amistades, intimidad, libertad y equilibrio emocional (11). De carácter multifactorial, la sobrecarga del cuidador involucra: el aislamiento social, sobrecarga de actividades tanto en casa como fuera de ella, alteración del comportamiento de los familiares que reciben cuidados, la idea de ser "responsables" exclusivos de su familiar, dificultades financieras, abandono de empleo por parte del cuidador, entre otras (12).

Frente a este escenario, la capacidad de agencia de autocuidado que desarrollen los cuidadores/as informales juega un rol funda- 
mental en el mantenimiento de la salud.

Basado en los conceptos de Dorothea Orem, autocuidado se refiere a la acción intencionada y calculada, que está condicionada por el conocimiento y repertorio de habilidades de un individuo, y se basa en la premisa que los individuos saben cuando necesitan ayuda y, por lo tanto, son conscientes de las acciones específicas que necesitan realizar, pudiendo escoger entre distintas opciones en sus conductas de autocuidado (13). Considera el concepto "auto" como la totalidad de un individuo, y el concepto "cuidado" como la totalidad de actividades que un individuo inicia para mantener la vida y desarrollarse de una forma que sea normal para él. Así, el autocuidado es la práctica de actividades que los individuos inician y realizan en su propio beneficio para el mantenimiento de la vida, la salud y el bienestar (13), justificando la función de enfermería en situaciones específicas en la que el individuo no puede cuidar de sí mismo, no tiene el conocimiento o no está motivado para hacerlo (14).

Los escritos de Orem se centran en la capacidad de las enfermeras y enfermeros de aportar al cuidado de las personas que no están en condiciones para desarrollarlas por sí mismas; sin embargo, hoy en día la gran mayoría de los cuidados a personas dependientes en sus domicilios los desarrollan los propios familiares, quienes poseen escasa preparación en el área y cuya motivación principal se centra en los afectos. Desde el sector salud, los profesionales brindan apoyo a estos cuidadores informales, pero es escaso, limitado principalmente por la demanda asistencial de los sistemas de atención primaria.

En Chile se ha comenzado a reconocer la presencia del cuidado informal. En forma incipiente, pero escasa, se han desarrollado estrategias públicas que lo abordan (15).

El Programa de Atención en Domicilio en Chile, desarrollado e implementado por los equipos de salud de los sistemas primarios, es una estrategia que evidencia la necesidad de cuidados en el hogar, frente a lo cual la ex Presidenta Michelle Bachelet, en el año 2006, integra para sus primeros 100 días de gobierno, la medida 6B. Esta medida apunta a dar apoyo a personas que sufren dependencia severa (4\% de la población mayor de 60 años) y están al cuidado de sus familiares, con el objetivo de mejorar la calidad del cuidado, aliviar a la familia cuidadora, disminuir la carga del cuidador/a y la institucionalización (16).

Esta medida se operacionaliza a través de las atenciones domiciliarias de los equipos de atención primaria, y consiste en un apoyo monetario, de pago mensual a la familia cuidadora, de acuerdo a una serie de criterios, entre otros: el nivel de dependencia y la condición socioeconómica (16).

Los profesionales de enfermería son los líderes de los equipos en las atenciones domiciliarias, y los cuidadores/as los principales aliados en la tarea del cuidar.

Insertos hoy en un modelo de atención con enfoque familiar y comunitario, interdisciplinario y transdisciplinario, en que el trabajo en equipo juega un rol fundamental en el mantenimiento y promoción de la salud, los cuidadores informales han surgido como un grupo de interés. De aquí se desprende la importancia de investigar lo que pasa con estos cuidadores/as, identificar sus características, el apoyo que reciben y cómo se cuidan, para luego desarrollar planes que permitan apoyarlos e intervenirlos a favor de su salud, razones que manifiestan la importancia en las acciones de enfermería, como ente gestor del cuidado.

Objetivos: Conocer el nivel de sobrecarga, el apoyo social percibido, la capacidad de agencia de autocuidado y otros factores relacionados, de los cuidadores de pacientes dependientes ingresados en el programa de atención domiciliaria del Servicio de Salud Municipal de la comuna de Tomé, en Chile, durante el año 2010. 


\section{MATERIAL Y MÉTODOS}

Estudio descriptivo transversal, que constituye el estudio piloto de un proyecto de investigación desarrollado en la comuna de Tomé, Chile, durante el año 2010. La muestra estuvo compuesta por 30 cuidadores/as informales principales, seleccionados en forma probabilística, sistemática y estratificada, extraídos de los listados de usuarios ingresados al programa de atención domiciliaria de los dos CESFAM en la comuna de la VIII Región, en Chile, con un 95\% de confianza y un error del $2 \%$.

La recolección de los datos se realizó por medio de visitas domiciliarias, durante el mes de agosto del año 2010, por medio de cuatro instrumentos: Cuestionario que evalúa características generales del cuidador informal, características del cuidado y del que recibe cuidados, diseñado por las autoras. Consta de 19 preguntas: de la 1 a la 10, las preguntas se enfocan en recolectar datos generales del cuidador; de la 11 a la 15 busca recolectar información sobre las características generales de la persona que recibe cuidados y de la pregunta 16 a la 19 busca recolectar datos sobre las características del cuidado. Cada pregunta posee su propia unidad de medida y se encuentra estructurada en respuestas cerradas, de alternativas o dicotómicas.

La Escala de sobrecarga de Zarit, instrumento que busca cuantificar el grado de sobrecarga que padecen los cuidadores/as de las personas dependientes, consta de 22 afir- maciones, en escala tipo Likert que va desde 0 (nunca), 1 (rara vez), 2 (algunas veces), 3 (bastantes veces) y 4 (casi siempre), con una puntuación global entre 0 y 88 puntos, en donde a mayor puntaje mayor nivel de sobrecarga (17).

La Escala Multidimensional de Apoyo Social Percibido de Zimet, compuesta por 12 ítemes, recoge información del apoyo social percibido por los individuos en tres áreas: familia, amigos y otros significativos. En este estudio se empleó la escala modificada, utilizada y validada en Santiago de Chile en un grupo de adultos mayores. Esta consta sólo de cuatro categorías, también divididas, en escala Likert: 1(casi nunca), 2 (a veces), 3 (con frecuencia) y 4 (siempre o casi siempre), cuyo puntaje va desde 12 a 48 puntos, en donde a mayor puntaje mayor apoyo social percibido (18).

La Escala de Apreciación de Agencia de Autocuidado (ASA) consta de 24 ítemes en formato de repuesta tipo likert de cinco alternativas, donde el número uno es totalmente en desacuerdo y el cinco es totalmente en acuerdo. En este estudio se empleó la escala modificada que consta de cuatro ítemes cuya puntuación va desde 1 (nunca), 2 (casi nunca), 3 (casi siempre), 4 (siempre), en un puntaje que va de 24 a 96 puntos, en que a mayor puntaje mayor nivel de agencia de autocuidado (19).

En relación a la determinación de la confiabilidad de los instrumentos, se estableció por medio de alfa de Cronbach (Tabla 1).

Tabla 1: Resultados pruebas de confiabilidad de instrumentos.

\begin{tabular}{|l|c|}
\hline Escala & Alfa de Cronbach \\
\hline Escala de Sobrecarga del Cuidador de Zarit & 0.7866 \\
\hline Escala Multidimensional de Apoyo Social Percibido de Zimet & 0.8432 \\
Subítem Familia & 0.8741 \\
Subítem Amigos & 0.9160 \\
Subítem Otros Significativos & 0.9284 \\
\hline Escala de Apreciación de Agencia de Autocuidado (ASA) & 0.8120 \\
\hline
\end{tabular}


En relación a los aspectos éticos del estudio, se solicitó previamente autorización a las direcciones de ambos CESFAM y al director del Servicio de Salud Municipal de la comuna de Tomé, además de la aprobación del comité de ética de la Universidad de Concepción. Con respecto a los cuidadores/ as informales, se solicitó su consentimiento informado para participar voluntariamente en el estudio, asegurando confidencialidad y anonimato de la información.

El análisis descriptivo de los datos se realizó a través del sistema estadístico SPSS versión 11.5 .

\section{RESULTADOS}

Entre las características biosociodemográficas del cuidador/a informal, se estableció que el $90 \%$ de los cuidadores/as informales son mujeres, cuyas edades fluctúan entre 37 y 86 años, con un promedio de 57 años. Según la situación de pareja, el 80\% de los cuidadores/as informales poseen pareja, el 76\% vive junto a su pareja, el $73.3 \%$ vive con hijos, el $66 \%$ con otros familiares y sólo el 10\% con amigos u otras personas.

En cuanto al nivel de escolaridad, el 6.7\% no presentan instrucción formal, el $43.3 \%$ educación básica incompleta, el 6.7\% educación básica completa, el 10\% educación media incompleta, el 30\% educación media completa y sólo el 3.3\% educación superior.

El 63\% de los cuidadores/as informales presenta alguna enfermedad por la cual se encuentra en tratamiento médico permanente, el $33.3 \%$ se dedican al cuidado de su madre, el $10 \%$ a un hijo/a, el $16.7 \%$ a un hermano/a, el $23.3 \%$ a su cónyuge, el 13.3\% a otro familiar y el 3.3\% a un amigo u otro. Cabe señalar que ningún cuidador que con- formó la muestra se encontraba al cuidado de su padre.

En cuanto al ingreso económico mensual familiar, sólo el $26.7 \%$ de los cuidadores/ as informales señala que era suficiente para satisfacer las necesidades básicas de la vida diaria y el $26.7 \%$ desarrolla algún trabajo remunerado.

El 33.7\% manifiesta su participación en alguna instancia grupal fuera de su casa.

En cuanto a las características de la persona cuidada, el 60\% corresponde a mujeres, cuya edad se encontraba entre 13 y 99 años, con un promedio de 71 años. Según el nivel de dependencia de acuerdo al Índice de Katz, el 43.3\% presenta una dependencia moderada y el $56.7 \%$ una dependencia severa, presentando en promedio 9 años en esta condición.

En relación a las características del cuidado, los cuidadores/as realizan esta labor hace 9 años como promedio, dedicando 17 horas del día a esta tarea y solo el $40 \%$ de los cuidadores recibe ayuda para efectuar el cuidado.

En cuanto a la complejidad de los cuidados brindados, el 90\% de los cuidadores/as realiza cuidados propios de la vida diaria, el $70 \%$ cuidados de menor complejidad, como administración de medicamentos, control de dieta, control de signos vitales y solo el $13.3 \%$ realiza cuidados de complejidad mayor, como curaciones, sondeos, administración de medicamentos inyectables.

Tanto la escala de sobrecarga como de apoyo social percibido mostraron una distribución normal, leptocúrtica con asimetría positiva. Por su parte la capacidad de agencia de autocuidado mostró una distribución normal, mesocúrtica simétrica.

La descripción de las variables sobrecarga del cuidador/a informal, apoyo social percibido y capacidad de agencia de autocuidado se muestran en la Tabla 2. 
Tabla 2: Estadísticos descriptivos de las variables sobrecarga del cuidador/a, apoyo social percibido y capacidad de agencia de autocuidado.

\begin{tabular}{|l|c|c|c|c|c|c|c|}
\hline Variable & Media & DS & Mediana & Moda & Rango & Mín. & Máx. \\
\hline Sobrecarga del cuidador & 33.8 & 13.8 & 32 & 14 & 53 & 14 & 7 \\
\hline $\begin{array}{l}\text { Capacidad de agencia } \\
\text { de autocuidado }\end{array}$ & 70.4 & 12.5 & 66 & 65 & 45 & 48 & 93 \\
\hline Apoyo social percibido & 24.6 & 9.2 & 24 & 13 & 34 & 12 & 46 \\
\hline
\end{tabular}

\section{DISCUSIÓN Y CONCLUSIÓN}

La revisión de la literatura da cuenta de estudios con relación directa en el tema de esta investigación y otros que hacen alusión a temas relacionados indirectamente.

Los estudios desarrollados, tanto en Chile como en el extranjero, establecen un perfil de los cuidadores/as informales similar al encontrado en la población en estudio, caracterizados por ser mujeres, entre 35 y 64 años de edad, con educación básica completa o incompleta, dedicados a las labores del hogar (5, 20-22). Sin embargo, un estudio desarrollado en Cuba, durante los años 2004-2005, establece que la mayoría de los cuidadores/ as informales, en esa población, poseen una preparación universitaria, sin experiencia previa en el cuidado (23), lo cual se puede relacionar con la realidad político-administrativa de ese país. Este mismo estudio demostró que la mayoría de los/as cuidadores/ as informales presentan alteraciones de salud, lo que es coincidente con los datos encontrados en este estudio, en que el 63\% de los encuestados presentaban alguna patología por la cual se encuentran en tratamiento.

El análisis muestral establece que los cuidadores familiares son, en su mayoría, hijas o cónyuges, situación distinta a la encontrada en un estudio desarrollado en Santiago de Chile, durante el año 2002, en un grupo de cuidadores de personas portadoras de VIH/ SIDA, en que los principales cuidadores fueron las madres (24).
En cuanto a la variable sobrecarga, los resultados mostraron que los/las cuidadores/ as presentaron en promedio 33.8 puntos, lo que se puede considerar como sobrecarga leve, similar a lo encontrado en México en que el $20 \%$ de los cuidadores presenta este nivel (21).

Respecto al apoyo social percibido, el resultado promedio fue de 24.3 puntos, lo que se puede considerar como bajo apoyo social. En cuanto a la variable autocuidado, no existen estudios desarrollados en este grupo de interés. Se han encontrado resultados relacionados con el tema, en poblaciones de pacientes con enfermedad coronaria, enfermedad crónica, VIH/ SIDA y/o riesgo cardiovascular, en los cuales se ha evaluado la capacidad de agencia de autocuidado, por medio de la escala de apreciación de agencia de autocuidado en su versión en español, demostrando su validez interna y externa (25-27). En este estudio establece un nivel de capacidad de agencia de autocuidado con un promedio de 70 puntos, el cual se puede considerar como una moderada capacidad de agencia de autocuidado.

De acuerdo a los datos emanados del estudio se establece que los cuidadores/as informales son en su mayoría mujeres, adultas, con pareja, con educación básica incompleta, con alguna patología por la cual reciban tratamiento médico permanente, responsables del cuidado de su madre y en menor medida de su cónyuge o pareja, no desarrollan algún trabajo remunerado, sin participación social y con percepción económica insuficiente 
para la satisfacción de las necesidades de la vida diaria, dedicando gran parte del día a la actividad de cuidar, sin recibir ayuda de terceros. En relación a la persona que recibe cuidados, en su mayoría son mujeres, adultas mayores, dependientes severas de larga data.

Los cuidadores/as informales presentan niveles de sobrecarga leve, con escasa percepción de apoyo social y desarrollo de moderada capacidad de agencia de autocuidado.

El nivel de sobrecarga del cuidador, el apoyo social percibido y la capacidad de agencia de autocuidado son indicadores que deben ser utilizados para la valoración de los/as cuidadores informales, en términos de las consecuencias que les produce la tarea de cuidar, para planificar actividades de apoyo a los/as cuidadores que permitan diseñar estrategias de ayuda y abordar en forma precoz dificultades, tanto en la calidad de vida como en la labor de cuidar.

Actualmente en el ámbito de enfermería se ha estudiado fuertemente este grupo de interés sanitario, para poder generar un perfil de los cuidadores/as y desarrollar estrategias de apoyo, que deben abordar más allá del ámbito de lo privado, sino en la generación de políticas públicas que trabajen esta problemática social y sanitaria.

A través de este estudio se permite dar una mirada de cómo estamos para luego generar estrategias de intervención efectivas en el área.

\section{REFERENCIAS}

1. Instituto Nacional de Estadísticas. Estadísticas Vitales, Informe Anual 2009. Santiago: INE; 2011.

2. Amnesty International. Informes anuales Chile 2009. [Internet]. New York: Amnesty International USA [citado 1 julio 2009]. Disponible en: http:// www.amnestyusa.org/annualreport_ es.php?id $=\operatorname{ar} \& y r=2009 \& c=$ CHL.
3. Departamento de Estadística e Información en Salud. Esperanza de vida al nacer (en años), según sexo, por quinquenios comprendidos entre los años 1950 y 2025 [Internet]. Santiago, Chile: DEISMINSAL [citado 9 junio 2010]. Disponible en: http://deis.minsal.cl/deis/ev/ esperanza_de_vida/index.asp.

4. Fondo Nacional de la Discapacidad (FONADIS)-Instituto Nacional de Estadísticas (INE). Primer Estudio Nacional de la Discapacidad [Internet]. Santiago de Chile: FONADIS; 2004. [citado 9 junio 2010]. Disponible en: http://www.ine.cl/canales/chile_ estadistico/encuestas_discapacidad/ discapacidad.php

5. Jofre V, Mendoza S. Toma de decisiones en salud en mujeres cuidadoras informales. Cienc. enferm. 2005; X (1): 3749.

6. Zabalegui A, Juando C, Izquierdo $M$, Gual P, González- Valentín A, Gallart A et al. Recursos y consecuencias de cuidar a las personas mayores de 65 años: una revisión sistemática. Gerokomos 2004; 15(4): 13-22.

7. García- Calvente M, Mateo- Rodríguez I, Eguiguren A. El Sistema de cuidados en clave de Desigualdad. Gac Sanit 2004; 18 (1): 132-139.

8. Barrón A, Sánchez E. Estructura social, apoyo social y salud mental. Psicothema 2001; 13 (1): 17-23.

9. Herrera E. Cuidadoras y cuidadores de personas dependientes y enfoque de género en Chile. Santiago: FONADIS; 2007.

10. Fondo Nacional de la Discapacidad (FONADIS)-Instituto nacional de Estadísticas (INE). Primer Estudio Nacional de la Discapacidad. Apartado Género [Internet]. Santiago de Chile: FONADIS; 2004. [citado 13 septiembre 2010]. Disponible en: http://www. gorecoquimbo.gob.cl/oremi/genero/ discapacidad/discapacidad_001.pdf 
11. González F, Graz A, Pitiot D, Podestá J. Sobrecarga del cuidador de personas con lesiones neurológicas. Revista del Hospital J.M. Ramos Mejias 2004; 9 (4): 1-22.

12. Mangini S. Vivenciando a sobrecarga ao vir-a-ser um cuidador familiar de pessoa com acidente vascular cerebral (AVC): análise do conhecimento. Rev Lat Am Enfermagem. 2004; 12(1): 115-121.

13. Bastías E, Sanhueza O. Conductas de Autocuidado y manifestaciones perimenopáusicas en mujeres de la comuna de Concepción, Chile. Cienc. enferm. 2004; $\mathrm{X}(1): 41-56$.

14. Martínez M, Escobar T, Soriano C. Autocuidado en mujeres en etapa de menopausia en Toluca, México. Esc. Anna Nery. 2008; 12 (1): 63-67.

15. Acosta E. El cuidado de las personas dependientes en las Políticas Públicas en Chile: ¿Quien cuidara de nosotros/as? Revista A- MERIKA 2009; 2(3).

16. División de Prevención y Control de Enfermedades, Departamento del Ciclo vital: Programa Adulto Mayor, Unidad de Discapacidad y Rehabilitación. Norma de Cuidados Domiciliarios de Personas que sufren de Discapacidad Severa; 2006.

17. Alvarez L, González A, Muñoz P. El cuestionario de sobrecarga del cuidador de Zarit. Cómo administrarlo e interpretarlo. Gac Sanit. 2008; 22(0): 618- 620.

18. Arechabala C, Miranda C. Validación de una escala de apoyo social percibido en un grupo de adultos mayores adscritos a un programa de hipertensión de la Región Metropolitana. Cienc. enferm. 2002; VIII (1): 49-55.

19. Velandia A, Rivera L. Confiabilidad de la Escala "Apreciación de Agencia de Autocuidado" (ASA), segunda versión en español, adaptada para la población colombiana. Av enferm. 2009; 27(1): 38-47. 20. Burgos P, Figueroa V, Fuentes M, Quija- da I, Espinoza E. Caracterización y nivel de conocimiento de cuidador informal de usuarios con dependencia severa adscritos al Centro de Salud Violeta Parra Chillán. Theoria 2008; 17 (2): 7-14.

21. Lara G, González A, Blanco L. Perfil del cuidador: sobrecarga y apoyo familiar e institucional del cuidador primario en el primer nivel de atención. Revista de Especialidades Médico-Quirúrgicas 2008; 13(4): 159-166.

22. Pérez A. El cuidador primario de familiares con dependencia: Calidad de vida, apoyo social y salud mental. [Internet]. Salamanca: Universidad de Salamanca, Programa: La enfermedad: su dimensión personal y condicionantes culturales; 2006. [citado 24 abril 2010]. Disponible en: http://www. laalamedilla.org/Investigacion/CD\%20 Tesis/Tesis \% $20 \mathrm{Anibal} /$ tesina $\% 20$ cuidadores06.pdf

23. Espin A. Caracterización psicosocial de cuidadores informales de adultos mayores con demencia. Rev Cub Salud Publica. 2008, 34(3): 1-12.

24. Cazanave A, Ferrer X, Castro S, Cuevas S. El Familiar cuidador de paciente con SIDA y la toma de decisiones en salud. Rev Chilena Infectol. 2005; 22(1): 51-57.

25. Bastidas C. Asociación entre la Capacidad de agencia de autocuidado y la adherencia a tratamientos farmacológicos y no farmacológicos en personas con alguna condición de enfermedad coronaria. Av enferm 2007; 25 (2): 65-75.

26. Rivera L, Díaz L. Relación entre la capacidad de agencia de autocuidado y los factores de riesgo cardiovascular. Cuad. - Hosp. Clín 2007, 52(2): 30-38.

27. Velandia A, Rivera L. Agencia de autocuidado y adherencia al tratamiento en personas con factores de riesgo cardiovasculares. Rev Salud Pública. 2009; 11 (4): 538-548. 